\title{
Uso da Valeriana officinalis em dietas de codornas japonesas na fase de postura
}

\author{
Rodrigo Antonio Gravena* \\ Rafael Henrique Marques \\ Janaina Della Torre Silva \\ Fabricio Hirota Hada \\ Vanessa Karla Silva \\ Danisio Prado Munari \\ Vera Maria Barbosa de Moraes \\ Departamento de Zootecnia, Universidade Estadual Paulista \\ CEP 14884-900, Jaboticabal, SP - Brasil \\ *Autor para correspondência \\ ragravena@hotmail.com
}

Submetido em 12/04/2009

Aceito para publicação em 29/08/2009

\section{Resumo}

Este estudo teve como objetivo avaliar o efeito da Valeriana officinalis sobre o estresse de codornas japonesas na fase postura, analisando-se parâmetros de desempenho, comportamental e fisiológico. Foram utilizadas 192 aves dos 43 aos 140 dias de idade, distribuídas em blocos casualizados e submetidas a quatro níveis de valeriana (controle, 250,500 e $750 \mathrm{mg} / \mathrm{kg}$ de ração) com oito repetições e seis aves por parcela. Foram avaliados parâmetros de desempenho (conversão alimentar por dúzia de ovos, conversão alimentar por massa de ovos, consumo de ração, peso dos ovos, porcentagem de postura e viabilidade), comportamentais (tempo em imobilidade tônica, observação focal e ferimentos corporais) e fisiológicos (níveis plasmáticos de corticosterona e relação heterófilo:linfócito). Os resultados obtidos na fase de postura demonstraram que a valeriana adicionada na dieta não foi capaz de reduzir o estresse das aves e não afetou o desempenho produtivo.

Unitermos: estresse, imobilidade tônica, níveis plasmáticos de corticosterona, relação heterófilo: linfócito, Valeriana officinalis

\section{Abstract}

Use of valerian in Japanese quail diet during the laying period. The effects of feeding levels of Valeriana officinalis on the performance, behavior and physiologic parameters of Japanese quails (Coturnix coturnix japonica) in the laying periods were evaluated. In the laying period, 192 quails were distributed in a randomized block design according to four nutrition plans (control, 250, 500 and 750mg of valerian $/ \mathrm{kg}$ of ration) with eight replicates and six birds per cage. Performance (feed intake, egg production, feed conversion per dozen eggs, feed conversion per egg $\mathrm{kg}$, egg weight and viability), behavior (body injury, tonic immobility 
and focal observation) and physiological parameters (heterophil/lymphocyte ratio and corticosterone blood plasma) were evaluated. The tested valeriana levels did not affect the performance, behavior or physiological parameters of Japanese quails in the laying phases.

Key words: corticosterone, Coturnix coturnix japonica, hererophil: lymphocyte ratio, tonic immobility, Valeriana officinalis

\section{Introdução}

A coturnicultura vem crescendo a cada ano e se tornando importante na geração de empregos principalmente aos pequenos produtores. Atualmente os grandes produtores de ovos comerciais estão também criando codornas em larga escala, por se tratar de uma atividade economicamente viável. No entanto, tem sido observado comportamento extremamente agressivo das aves devido ao elevado nível de estresse, causando ferimentos por bicadas nas penas, agressão, desvio social e depressão (Mills e Faure, 1990; Jones, 1996), podendo afetar tanto a saúde como a produtividade de forma direta ou indireta (Mills e Faure, 1990).

Aves expostas a predadores ou a situações novas apresentam comportamento de medo levando ao estado de hipnose ou imobilidade tônica que persiste por alguns segundos até horas. Este estado de estresse pode ser avaliado pela tomada de tempo em imobilidade tônica (TIT) do animal (Figueiredo et al., 2003).

As aves quando submetidas a situações de estresse passam por processos fisiológicos que aumentam a liberação de hormônios corticotróficos, reduzindo a quantidade de linfócitos, aumentando a relação heterófilo:linfócito (H:L) (Macari et al., 2002). Submetendo aves a diferentes situações de estresse, como elevados níveis de amônia no ar, choques elétricos e temperaturas elevadas, McFarlane e Curtis (1989) observaram aumento na relação H:L e concluíram que este parâmetro é uma medida eficiente na avaliação da intensidade de estresse das aves.

Uma alternativa visando diminuir a agressividade de codornas e obviamente melhorar o bem estar, seria o uso de fitoterápicos, porém, é um assunto que ainda precisa de estudos, pois se têm poucos parâmetros para a sua utilização em codornas.

Dentre vários fitoterápicos existentes, o escolhido para este estudo foi a Valeriana officinalis, também conhecida como amantila, bardo selvagem, erva gata, valaricana e badarina. É uma planta medicinal eficaz contra ansiedade, angústia, indutor do sono e leves desequilíbrios do sistema nervoso (Psiqweb, 2004), sendo comprovado seu efeito sedativo em humanos com dificuldades de dormir, provocando redução no tempo requerido para dormir, menor quantidade de movimentos na cama e ausência de ressaca matinal em relação ao grupo placebo (Leathwood e Chauffard, 1985).

Como até o momento pouco se sabe sobre os efeitos de fitoterápicos sobre o desempenho e agressividade das aves, o presente trabalho teve como objetivo estabelecer os níveis de inclusão de Valeriana officinalis na ração de codornas como modulador de estresse.

\section{Materiais e Métodos}

Este trabalho foi realizado no Setor de Avicultura do Departamento de Zootecnia da Faculdade de Ciências Agrárias e Veterinárias de Jaboticabal, em galpão convencional para codornas.

Foram utilizadas 192 codornas não debicadas, vacinadas contra Newcastle, com 42 dias de idade distribuídas em delineamento em blocos ao acaso em gaiolas para controlar o efeito inicial do peso, sendo submetidas a 4 tratamentos (controle; 250; 500 e $750 \mathrm{mg}$ de Valeriana officinalis/kg de ração), com 8 repetições e 6 aves por parcela, mais 24 aves de reposição para substituir as aves mortas, recebendo os mesmos tratamentos com a finalidade de submeter estas aves a uma mesma densidade por área.

$\mathrm{O}$ extrato de valeriana adicionado na dieta das aves experimentais foi obtido pela secagem e moagem do rizoma da planta. Este produto em forma de pó contem linarina e flavonóides como princípios ativos. As rações foram isoprotéicas e isoenergéticas, e seguiram as tabelas de composição de ingredientes de Rostagno et 
al. (2005) e as exigências nutricionais foram baseadas nas recomendações feitas por Murakami et al. (1993) e o NRC (1994). A Valeriana officinalis foi adicionada aos tratamentos nas concentrações especificadas na Tabela 1.

O primeiro ciclo de postura teve início quando as aves atingiram $15 \%$ de postura com 42 dias de idade, momento em que se estabeleceu o programa de luz, onde o período de luz foi aumentando gradativamente até atingir 17 horas diárias. Ao final de cada ciclo foram pesadas as sobras de ração e os ovos de cada parcela, sendo estes pesados nos 3 últimos dias do ciclo, para se obter a conversão alimentar, o consumo de ração e o peso médio dos ovos.

Foram avaliados os parâmetros de desempenho: consumo de ração (g/dia), conversão alimentar (consumo de ração/dúzia e kg de ovos), viabilidade, porcentagem de postura e peso dos ovos; tempo em imobilidade tônica (TIT); níveis plasmáticos de corticosterona, relação H:L, observação focal das atividades realizadas e ferimentos corporais.

OTIT consiste na tomada de tempo, em segundos, em que a codorna permanece imóvel quando repentinamente ela é colocada em decúbito dorsal em uma superfície plana, fazendo-se leve pressão sobre o peito durante alguns segundos na tentativa de imobilizar a ave (Heiblum et al., 1998).

Com o uso de seringa com EDTA, uma pequena amostra de sangue obtida por punção da veia braquial foi utilizada para a confecção das lâminas para as contagens de H:L em microscópio óptico (Campo e Dávila, 2002). Utilizando-se outra seringa com heparina foram coletadas das mesmas aves e da mesma maneira, $0,5 \mathrm{~mL}$ de sangue por ave, que foram mantidos sob refrigeração durante a coleta e centrifugados para a extração do plasma mantido congelado a $-20^{\circ} \mathrm{C}$ para posterior análise dos níveis de corticosterona utilizando-se kits próprios para radiomunoensaio (Malheiros et al., 2003).

Nas observações focais das atividades realizadas pelas aves, foram analisadas as atividades agressivas e não agressivas, descritas no etograma, que foram feitas pelas observações focais diretas e contínuas do comportamento social das aves. As observações focais foram feitas duas vezes por semana durante todo o período experimental (42 aos 140 dias de idade das aves), sendo o tempo de observação de cinco minutos por parcela, onde o observador registrou todos os comportamentos e atividades de todas as aves. As observações foram feitas sempre no período da manhã e no mesmo horário.

TABELA 1: Composição percentual e calculada das rações fornecidas às aves na fase de postura de acordo com os tratamentos.

\begin{tabular}{|c|c|c|c|c|}
\hline \multirow[t]{2}{*}{ Ingredientes $(\%)$} & \multicolumn{4}{|c|}{$\begin{array}{l}\text { Valeriana officinalis } \\
\text { (mg/ kg de ração) }\end{array}$} \\
\hline & $\mathbf{0}$ & 250 & 500 & 750 \\
\hline Milho & 63,507 & 63,482 & 63,457 & 63,432 \\
\hline Farelo de soja & 27,622 & 27,622 & 27,622 & 27,622 \\
\hline Fosfato bicálcico & 2,602 & 2,602 & 2,602 & 2,602 \\
\hline Calcário calcítico & 4,864 & 4,864 & 4,864 & 4,864 \\
\hline $\mathrm{Sal} / \mathrm{NaCl}$ & 0,400 & 0,400 & 0,400 & 0,400 \\
\hline $\begin{array}{l}\text { Suplemento vitamínico } \\
\text { e mineral }{ }^{1}\end{array}$ & 0,500 & 0,500 & 0,500 & 0,500 \\
\hline Lisina & 0,336 & 0,336 & 0,336 & 0,336 \\
\hline DL.Metionina & 0,169 & 0,169 & 0,169 & 0,169 \\
\hline Valeriana & 0 & 0,025 & 0,050 & 0,075 \\
\hline \multirow[t]{2}{*}{ Total } & 100 & 100 & 100 & 100 \\
\hline & \multicolumn{4}{|c|}{ Composição calculada } \\
\hline Proteína bruta (\%) & 18 & 18 & 18 & 18 \\
\hline $\begin{array}{l}\text { Energia Metabolizável } \\
(\mathrm{kcal} / \mathrm{kg})\end{array}$ & 2800 & 2800 & 2800 & 2800 \\
\hline Cálcio (\%) & 2,50 & 2,50 & 2,50 & 2,50 \\
\hline Fósforo disponível (\%) & 0,55 & 0,55 & 0,55 & 0,55 \\
\hline Lisina $(\%)$ & 1,30 & 1,30 & 1,30 & 1,30 \\
\hline Metionina + Cistina $(\%)$ & 0,76 & 0,76 & 0,76 & 0,76 \\
\hline
\end{tabular}

${ }^{1}$ Suplemento vitamínico e mineral - Composição/kg do produto: Ácido fólico, 61,75mg; Biotina, 25mg; Colina, 60000mg; Niacina, 2475mg; Pantotenato de cálcio, 712,5mg; Vit. A, 1562500 UI; Vit. $\mathrm{B}_{1}, 370 \mathrm{mg}$; Vit. $\mathrm{B}_{12}, 5000 \mathrm{mcg}$; Vit. $\mathrm{B}_{2}, 850 \mathrm{mg}$; Vit. $\mathrm{B}_{6}, 247,5 \mathrm{mg}$; Vit. $D_{3}$, 625000 UI; Vit. E, 3125mg; Vit. K, 245mg; Cobre, $1875 \mathrm{mg}$; Iodo, 126,5mg; Manganês, 11437,5mg; Selênio, 57mg; Zinco, $15057 \mathrm{mg}$; Metionina, 350g; Antioxidante, 100mg.

Etograma:

a) Comportamentos agressivos:

- Monta: momento em que uma ave monta (pisa) sobre a outra ave. 
- Bica: ocorre quando uma ave bica a outra ave no corpo ou na cabeça.

- Empurra: movimento em que a ave mais agitada tromba com outra ave.

- Agitada: momento em que a ave se agita e corre por toda a gaiola.

b) Comportamentos não agressivos

- Ócio: momento em que a ave fica deitada no piso da gaiola.

- Coça: momento em que a ave se coça tanto com as patas como com o bico.

- Come

- Bebe

- Defeca

Os ferimentos corporais foram avaliados pela presença ou não de lesões nas diferentes partes do corpo (dorso, cauda, asas e cabeça) das aves, individualmente, ao final de cada ciclo de postura das aves, após a avaliação do tempo em imobilidade tônica, durante o período da manhã.

As análises estatísticas não paramétricas de atividades realizadas e ferimentos corporais das aves foram feitas pela comparação de médias pelo teste de Qui-quadrado a $5 \%$ de probabilidade. Para os outros resultados obtidos, foi utilizado o procedimento GLM do SAS $^{\circledast}$ (Statistical Analysis System, 1995). Para se verificar a significância entre as médias dos tratamentos foi utilizado o teste de Tukey a 5\% de probabilidade.

\section{Resultados e Discussão}

Analisando-se os resultados de desempenho obtidos durante a fase de postura, observou-se que a valeriana não foi capaz de atuar de forma significativa sobre os parâmetros avaliados (Tabela 2).

Estes resultados de desempenho concordam com os obtidos por Matos (2006) e Marques (2007). Marques (2007) avaliou o desempenho de codornas que receberam dietas suplementadas com níveis crescentes de camomila, e Matos (2006) testou três níveis de probióticos, prebióticos e fitoterápicos (extrato de babosa e confrei) adicionados à dieta das aves.

Em estudo com codornas na fase de postura alimentadas com dietas suplementadas com diferentes níveis de passiflora, Silva (2006) verificou diferenças significativas entre os tratamentos para conversão alimentar (dz/kg), sendo o melhor resultado obtido para as aves alimentadas com ração contendo $250 \mathrm{mg}$ de passiflora/kg de ração.

Os resultados estatísticos demonstraram que não houve diferenças significativas $(P>0,05)$ entre os tratamentos para os níveis plasmáticos de corticosterona, relação H:L e TIT (Tabela 3).

Nos resultados estatísticos do TIT, pode-se observar que o valor do coeficiente de variação foi elevado, e

TABELA 2: Resultados de consumo diário de ração (CR), peso dos ovos (PO), conversão alimentar (CA - kg/ $\mathrm{kg}$ de ovos), conversão alimentar por dúzia (CD $-\mathrm{kg} / \mathrm{dz}$ de ovos), viabilidade (V) e porcentagem de postura (PP) das aves durante a fase de postura.

\begin{tabular}{ccccccc}
\hline Valeriana $(\mathbf{m g})$ & $\mathbf{C R}(\mathbf{g})$ & $\mathbf{P O}(\mathbf{g})$ & $\mathbf{C A}(\mathbf{k g})$ & $\mathbf{C D}(\mathbf{d u ́ z i a})$ & $\mathbf{V}(\mathbf{\%})$ & $\mathbf{P P}(\mathbf{\%})$ \\
\hline $\mathbf{0}$ & 26,58 & 11,42 & 2,70 & 0,37 & 99,83 & 86,81 \\
$\mathbf{2 5 0}$ & 25,87 & 11,51 & 2,80 & 0,39 & 99,78 & 81,82 \\
$\mathbf{5 0 0}$ & 26,50 & 11,34 & 2,76 & 0,38 & 99,95 & 85,21 \\
$\mathbf{7 5 0}$ & 26,51 & 11,42 & 2,69 & 0,37 & 99,82 & 86,22 \\
\hline $\mathbf{C V} \mathbf{( \% )}$ & 1,45 & 2,18 & 7,26 & 6,89 & 0,12 & 6,58 \\
$\mathbf{D M S}$ & 0,90 & 0,34 & 0,27 & 0,03 & 0,17 & 7,80 \\
$\mathbf{F}$ & $2,22^{\text {ns }}$ & $0,64^{\text {ns }}$ & $0,51^{\text {ns }}$ & $0,85^{\text {ns }}$ & $2,41^{\text {ns }}$ & $1,27^{\text {ns }}$ \\
\hline
\end{tabular}

ns $=$ não significativo $(\mathrm{P}>0,05)$. 
isso pode ser explicado pela variação individual de cada codorna.

Foram observados resultados semelhantes de TIT em estudo feito por Marques (2007), que utilizou níveis crescentes de camomila na dieta das codornas na fase de postura, com a finalidade de reduzir o estresse e a agressividade das aves. Discordando dos resultados obtidos neste estudo, Matos (2006) avaliou o TIT de codornas na fase de postura submetidas a dietas com níveis crescentes de prebióticos, probióticos e fitoterápicos, com o intuito de reduzir a agressividade e estresse das aves, e concluiu que o tratamento com o maior nível dos produtos foi eficaz em reduzir o TIT em relação ao tratamento controle. Da mesma maneira, Silva (2006) verificou menor duração no TIT de aves suplementadas com passiflora em relação às aves não suplementadas.

TABELA 3: Resultados dos níveis plasmáticos de corticosterona (NC), relação heterófilo: linfócito (H:L) e tempo em imobilidade tônica (TIT) das aves no período de postura.

\begin{tabular}{cccc}
\hline Valeriana (mg) & NC (ng/ml) & H:L & TIT $(\mathbf{s e g})$ \\
\hline $\mathbf{0}$ & 69,34 & 1,01 & 7,22 \\
$\mathbf{2 5 0}$ & 67,52 & 0,97 & 5,64 \\
$\mathbf{5 0 0}$ & 72,33 & 1,71 & 4,11 \\
$\mathbf{7 5 0}$ & 60,64 & 0,94 & 6,83 \\
\hline $\mathbf{C V}(\boldsymbol{\%})$ & 35,71 & 53,20 & 78,63 \\
$\mathbf{D M S}$ & 33,57 & 0,86 & 6,52 \\
$\mathbf{F}$ & $0,34^{\mathrm{ns}}$ & $2,89^{\text {ns }}$ & $0,71^{\text {ns }}$ \\
\hline
\end{tabular}

${ }^{\mathrm{ns}}=$ não significativo $(\mathrm{P}>0,05)$.

Semelhantes aos resultados obtidos neste estudo, em experimentos com codornas na fase de postura, no qual se avaliou a relação $H: L$ das aves, não houve efeito significativo devido a inclusão de diferentes níveis de passiflora (Silva, 2006) e camomila (Marques, 2007) na dieta das aves. Por outro lado, contrariando os resultados obtidos, quando da utilização da passiflora os níveis plasmáticos de corticosterona foram menores nas codornas que receberam passiflora em relação ao tratamento controle (Silva, 2006). A utilização de camomila, como neste trabalho, não apresentou diferenças significativas entre os tratamentos (Marques, 2007).

Os resultados de TIT, relação $\mathrm{H}: \mathrm{L}$ e níveis plasmáticos de corticosterona obtidos neste estudo comprovam que o fitoterápico não foi capaz de reduzir o estresse das aves nos níveis adicionados nas dietas, o que pode explicar o motivo pelo qual os resultados de desempenho não apresentaram diferença significativa.

Observando-se os resultados da Tabela 4, concluise que a valeriana não foi capaz de exercer efeito sedativo ou ansiolítico sobre as aves suplementadas com estas dietas, não havendo redução expressiva dos ferimentos corporais. Apenas as aves que receberam $250 \mathrm{mg}$ de valeriana/kg de ração apresentaram menores incidências de lesões no corpo, porém com valores estatisticamente iguais ao tratamento controle. As aves submetidas aos tratamentos com 500 e $750 \mathrm{mg}$ de valeriana/kg de ração não diferiram estatisticamente em relação aos ferimentos corporais, porém diferiram do controle e $250 \mathrm{mg}$ de valeriana/kg de ração, tendo maior número de aves com lesão. Isto leva a acreditar que houve efeito contrário ao esperado, ou seja, houve um maior grau de excitação das aves alimentadas com estes níveis do fitoterápico, o que induz a constatar que há um nível ideal para reduzir a agressividade das aves entre $250 \mathrm{mg}$ e $500 \mathrm{mg}$ de valeriana/kg de ração. Não houve diferença estatística entre os tratamentos pelo teste do Qui-quadrado ( $\mathrm{P}=0,67$ e valor do qui-quadrado $=1,54)$ para lesões na cabeça.

TABELA 4: Ferimentos corporais das aves na fase de postura.

\begin{tabular}{ccc}
\hline Valeriana (mg) & $\begin{array}{c}\text { Sem lesão } \\
(\%)\end{array}$ & $\begin{array}{c}\text { Com lesão } \\
(\%)\end{array}$ \\
\hline $\mathbf{0}$ & 95,8 & $4,2 \mathrm{~A}$ \\
$\mathbf{2 5 0}$ & 99,4 & $0,6 \mathrm{~A}$ \\
$\mathbf{5 0 0}$ & 75,9 & $24,1 \mathrm{~B}$ \\
$\mathbf{7 5 0}$ & 76,8 & $32,2 \mathrm{~B}$ \\
\hline Valor do qui-quadrado & \multicolumn{2}{c}{20,74} \\
\hline Probabilidade & \multicolumn{2}{c}{0,0001} \\
\hline
\end{tabular}

Médias seguidas por letras iguais não diferem entre si pelo teste do Qui-quadrado ( $\mathrm{P}>0,05)$.

Em estudos feitos com codornas na fase de postura recebendo dietas suplementadas com níveis crescentes 
de prebióticos, probióticos e fitoterápicos (Matos, 2006) e camomila (Marques, 2007), foram avaliados os ferimentos corporais (cabeça e corpo) das aves e concluiu-se que os fitoterápicos estudados isoladamente não foram capazes de reduzir a agressividade das aves.

Observando os resultados da Tabela 5, pode-se concluir que para os parâmetros de comportamento avaliados não houve influência do fitoterápico. Estes resultados concordam parcialmente com os obtidos em outros estudos, onde codornas na fase de postura que receberam níveis crescentes de prebióticos, probióticos e fitoterápicos (Matos, 2006) e camomila (Marques, 2007) não tiveram influência expressiva no comportamento das aves, sendo os fitoterápicos avaliados de forma isolada, ineficientes em reduzir a agressividade.

TABELA 5: Comportamentos das aves na fase de postura submetidas a diferentes níveis de valeriana na dieta expressos em valores percentuais de tempo médio.

\begin{tabular}{clcccc}
\hline \multirow{2}{*}{ Comportamento } & \multicolumn{5}{c}{ Valeriana (mg) } \\
\cline { 2 - 6 } & Monta & 11,12 & 10,07 & 11,96 & 12,54 \\
\multirow{4}{*}{ Agressivos } & Bica & 3,97 & 4,85 & 4,42 & 6,05 \\
& Empurra & 0,90 & 0,81 & 1,00 & 0,82 \\
& Agitada & 13,02 & 13,02 & 9,86 & 13,95 \\
\hline Come & 41,38 & 40,17 & 40,74 & 36,50 \\
Não agressivos & Oécio & 16,12 & 13,68 & 14,27 & 14,00 \\
& Coça & 8,74 & 6,85 & 7,12 & 5,56 \\
& Defeca & 1,26 & 1,40 & 1,52 & 1,56 \\
\hline Total & 100 & 100 & 100 & 100 \\
\hline Valor qui-quadrado & \multicolumn{5}{c}{3,42} \\
\hline Probabilidade & \multicolumn{5}{c}{1,00} \\
\hline
\end{tabular}

Silva (2006) observou que a adição de passiflora na dieta de codornas na fase de postura foi capaz de diminuir a agressividade das aves, reduzindo a interação entre as aves e o número médio de montas, proporcionando bem estar, porém, não havendo diferenças significativas para o número médio de bicadas entre as aves.
A partir dos resultados obtidos, concluiu-se que os níveis crescentes de valeriana adicionados à dieta das codornas não foram capazes de exercer os efeitos benéficos esperados sobre os parâmetros de desempenho, fisiológicos e comportamentais.

\section{Agradecimentos}

À Fundação de Amparo a Pesquisa do Estado de São Paulo (FAPESP) pelo apoio financeiro. À Prof ${ }^{a}$ Dra $^{\mathrm{a}}$ Alice Eiko Murakami da Universidade Estadual de Maringá (UEM) pela doação das codornas.

\section{Referências}

Campo, J. L.; Dávila, S. G. 2002. Influence of mating ratio and group size on indicators of fearfulness and stress hens and cocks. Poultry Science, 81: 1099-1103.

Figueiredo, G.; Queiroz, S. A.; Tanaka, A. L. R.; Bruneli, F. A. T.; Tholon, P. 2003. Avaliação do estresse em perdizes brasileiras (Rhynchotus rufescens) criadas cativeiro mediante imobilidade tônica. Anais do Congresso de Iniciação Científica da UNESP, Marília, Brasil, p.15.

Heiblum, R.; Aizenstein, O.; Gvaryahu, G.; Voet, H.; Robinson, B.; Snapir, N. 1998. Tonic immobility and open field responses in domestic fowl chicks during the first week of life. Applied Animal Behaviour Science, 60: 347-357.

Jones, R. B. 1996. Fear and adaptability in poultry: Insights, implications and imperatives. World's Poultry Science Journal, 52: 131-174.

Leathwood, P. D.; Chauffard, F. 1985. Aqueous extract of valerian reduces latency to fall asleep. Planta Médica, 2: 144-148.

Macari, M.; Furlan, R.; Gonzales, E. 2002. Fisiologia aviária aplicada a frangos de corte. Ed. Funep, Jaboticabal, Brasil, 20pp. Malheiros, R. D.; Moraes, V. M. B.; Collin, A.; Decuypere, E.; Buyse, J. 2003. Free diet selection by broilers as influenced by dietary macronutrients ratio and corticosterone supplementation. 1. Diet selection, organ weights, and plasma metabolites. Poultry Science, 82: 123-131.

Marques, R. H. 2007. Uso da camomila (Matricaria chamomila) em dieta de codornas japonesas. Trabalho de Graduação, Faculdade de Ciências Agrárias e Veterinárias, Universidade Estadual Paulista, Brasil, 49pp.

Matos, A. S. 2006. Uso de ESTIBION BÁSICO PLUS ${ }^{\circledR}$ na dieta de codornas japonesas na fase de postura. Trabalho de Graduação, Faculdade de Ciências Agrárias e Veterinárias, Universidade Estadual Paulista, Brasil, 42pp.

McFarlane, J. M.; Curtis, S. E. 1989. Multiple concurrent stressors in chickens. Effects on plasma corticosterone and the heterophil:lymphocyte ratio. Poultry Science, 68: 522-527.

Mills, A. D.; Faure, J. M. 1990. Panic and hysteria in domestic fowl: A review. In: Zayan, R. \& Dantzer, R. (Eds). Social stress 
in domestic animals. Kluwer Academic Publisher, Dordrecht, Netherlands, p.248-272.

Murakami, A. E.; Moraes, V. M. B.; Ariki, J.; Junqueira, O. M.; Kronka, S. M. 1993. Níveis de proteína e energia em rações para codornas (Coturnix coturnix japonica) em postura. Revista Brasileira de Zootecnia, 22(4): 541-551.

National Research Council - NRC. 1994. Nutrient requirements of poultry. National Academy Press, Washington, USA, 156pp.
Psiqweb. 2004. Disponível em <http://www.psiqweb.med.br/ farmaco/fitot.html>. Acesso em 20 dezembro de 2004.

Rostagno, H. S.; Albino, L. F. T.; Donzele, J. L.; Gomes, P. C.; Oliveira R. F.; Lopes, D. C.; Ferreira, A. S.; Barreto, S. L. T. 2005. Tabelas brasileiras para aves e suínos: Composição de alimentos e exigências nutricionais. UFV, Viçosa, Brasil, 186pp.

Silva, J. D. T. 2006. Uso da Passiflora alata na alimentação de codornas nas fases de recria e postura. Dissertação de Mestrado Universidade Estadual Paulista, Brasil, 57pp.

Statistical Analysis Systems Institute. 1995. User's guide. Version $6,4^{\text {th }}$ ed. SAS ${ }^{\circledR} / \mathrm{STAT}$, SAS Institute Inc.,Cary, USA, 365pp. 